\title{
Endoscopic ultrasound-based transluminal cholecystolithotomy: the retrievable anchor assisted procedure
}

Nan Ge

Shingling Hospital, China medical university https://orcid.org/0000-0002-5764-7054

Jinlong Hu

Shengjing Hospital of China Medical University

Fei Yang

Shengjing Hospital of China Medical University

Fan Yang

Shengjing Hospital of China Medical University

Kai Zhang

Shengjing Hospital of China Medical University

Siyu Sun ( $\square$ sunsy@sj-hospital.org )

https://orcid.org/0000-0002-7308-0473

\section{Research article}

Keywords: Cholecystolithotomy; endoscopic ultrasound; retrievable anchor; lumen apposing metal stent

Posted Date: July 16th, 2019

DOI: https://doi.org/10.21203/rs.2.11425/v1

License: (c) (i) This work is licensed under a Creative Commons Attribution 4.0 International License.

Read Full License 


\section{Abstract}

Background EUS guided gallbladder drainage has gradually emerging as an option among patients with cholecystitis. Stent bridged endoscope intervention in the gallbladder was further introduced. The aim of this study was to assess the effectiveness and safety of the ECE-LAMS and this newly designed retrievable puncture anchor applied in the transmural Cholecystolithotomy. Method We retrospectively reviewed consecutive patients undergoing EUS-guided transmural cholecystoscopic therapy. The main outcome measures for the EUS-GBD procedure were techniques success and adverse events, which were also compared between guided assisted group and retrievable anchor assisted group. The main outcome measures for the transmural cholecystoscopy after EUS-GBD were the stent indwelling time, clinical success and adverse events. Results A total of 24 (15 female) patients with gallstones were received the transluminal Cholecystolithotomy. Mean age is $36.08 \pm 13.69$ years old. The successful rate of the anchor assisted group was high than the guidewire assisted group ( $92.9 \%$ Vs $70 \%, \mathrm{P}=0.07)$. On the average of 12.25 \pm 2.77 (range 8-17) days after the initial BD drainage procedure, 20 patients received the per-oral cholecystoscopy via the fistula. Fistula broken was found in 2 patients in which the per-oral cholecystoscopy was applied on the ninth day after the EUS-GBD. During the follow-up period (mean $314.2 \pm 213.7$ days) no GB stones recurrence or other long term complications reported. Conclusion In conclusion, we have demonstrated that EUS-assisted per-oral cholecystoscopy technique with ECE-LMAS is an efficient and safe procedure. For the EUS-GBD procedure, retrievable anchor could significantly increase the success rate compared with the guidewire.

\section{Abstract}

\section{Background}

EUS guided gallbladder drainage has gradually emerging as an option among patients with cholecystitis. Stent bridged endoscope intervention in the gallbladder was further introduced. The aim of this study was to assess the effectiveness and safety of the ECE-LAMS and this newly designed retrievable puncture anchor applied in the transmural Cholecystolithotomy.

\section{Method}

We retrospectively reviewed consecutive patients undergoing EUS-guided transmural cholecystoscopic therapy. The main outcome measures for the EUS-GBD procedure were techniques success and adverse events, which were also compared between guided assisted group and retrievable anchor assisted group. The main outcome measures for the transmural cholecystoscopy after EUS-GBD were the stent indwelling time, clinical success and adverse events.

\section{Results}


A total of 24 ( 15 female) patients with gallstones were received the transluminal Cholecystolithotomy. Mean age is $36.08 \pm 13.69$ years old. The successful rate of the anchor assisted group was high than the guidewire assisted group (92.9\% Vs $70 \%, P=0.07$ ). On the average of $12.25 \pm 2.77$ (range 8-17) days after the initial BD drainage procedure, 20 patients received the per-oral cholecystoscopy via the fistula. Fistula broken was found in 2 patients in which the per-oral cholecystoscopy was applied on the ninth day after the EUS-GBD. During the follow-up period (mean 314.2 213.7 days) no GB stones recurrence or other long term complications reported.

\section{Conclusion}

In conclusion, we have demonstrated that EUS-assisted per-oral cholecystoscopy technique with ECELMAS is an efficient and safe procedure. For the EUS-GBD procedure, retrievable anchor could significantly increase the success rate compared with the guidewire.

\section{Keywords:}

Cholecystolithotomy; endoscopic ultrasound; retrievable anchor; lumen apposing metal stent

\section{Take-home message}

\section{What is already known:}

After nearly 10 years' development, EUS-GBD has become an attractive alternative for managing acute cholecystitis in high-risk patients.

Formation of fistulous tract after EUS-GBD facilitates the entry of endoscope inside GB to perform endoscopic lithotomy and polypectomy

\section{What's new?}

Newly developed devices like metal stent with hot delivery system and retrievable anchor could further facilitate the procedure.

The stent indwelling time should no less than 10 days before the following endoscopic trans-mural operations

\section{Background}


Endoscopic ultrasound guided gallbladder drainage (EUS-GBD) is gradually emerging as an option among patients who are not eligible for surgical interventions because of advanced malignancy or high surgical risk ${ }^{1}$. However, after EUS-GBD most of the patients were followed with a wait-and-see strategy until clinical symptoms or stent-related adverse events developed. For gallstones and gallbladder polyps, can we take further steps besides just "wait and see"? Besides drainage, is there any endoscopic approach available? The metal stent has already established a bridge for stomach or duodenum to the gallbladder. Through this bridge, we may accomplish endoscopic cholecystolithotomy or polypectomy.

In 2012, we started the animal study and proved the feasibility and safety of EUS guided trans gastric cholecystolithotomy ${ }^{2}$. In 2015 we reported the initial attempt of EUS-assisted transduodenal Cholecystolithotomy ${ }^{3}$ and then followed with retrospective studies ${ }^{4}$, which all verified the feasibility of the technique. At that time, the procedure was accomplished by the lumen apposing metal stent (LAMS) without hot deliver system which was assisted by the guidewire. In 2018, we reported the initial animal study of the newly developed electrocautery-enhanced delivery of LAMS (ECE-LAMS) and a retrievable puncture anchor applied in the transduodenal Cholecystolithotomy, which significantly simplified the procedure and increased the success rate of EUS-GBD ${ }^{5}$. So far, there was few study on the clinical evaluation of the retrievable anchor assisted deployment of the ECE-LAMS. In this study, we evaluated and compared the efficiency of anchor assisted EUS-based transluminal Cholecystolithotomy via ECELAMS with the guidewire assisted approach.

\section{Methods}

\section{Patients}

Form Jan 2017 to Dec 2018, patients received the EUS transluminal cholecystolithotom (assisted by guidewire or retrievable puncture anchor) in Shengjing Hospital was reviewed. The including criteria: 1. patients with gallstones and recurrent cholecystitis 2 . Patients refused the cholecystectomy 3 . The gallbladder still has the satisfied function. Exclusion criteria were 1. gallbladder atrophy. 2. coagulopathy and other sever comorbidity. The protocol to perform retrospective revision of the cases was approved by the Medical Ethics Committees. All patients gave their informed consent before the use the procedure.

\section{Main coutcome measurement}

The main coutcome measures for the EUS-GBD procedure were techniques success, clinical effectiveness, and adverse events, which were also compared between guided assisted group and retrievable anchor assisted group. The main outcome measures for the tansmural cholecystoscopic therapy were the strent indwelling time, clinical success and adverse events.

\section{The study device}


The device used in in this study is a through the scope LAMS with an electrocautery deliver system $(12 \mathrm{~mm} / 25 \mathrm{~mm}, 16 \mathrm{~mm} / 35 \mathrm{~mm}$; Micro-Tech/Nan Jing Co, Ltd). The stent is with wide flanges on both ends that provide anchoring within the gallbladder. The stent is delivered through a $9 \mathrm{~F}-10.5 \mathrm{~F}$ catheter. In some patients, the retrievable puncture anchor (Figure 1) (Vedkang Inc., Changzhou, China) was applied to anchor the GB during the ECE-LAMS puncturing.

\section{The procedure}

There are two parts of the treatment for gallbladder stone, which are EUS-guided GBD at the first time and followed with cholecystolithotomy or polypectomy when the fistula between the duodenal(stomach) and the GB formed. All the patients were under general anesthesia and in the lie-down position during operation. After the cholecystolithotomy procedure, the patients were followed the protocol of Minimally invasive endoscopic gallbladder preserved cholecystolithotomy guideline in China (2015) and were suggested periodical US follow-up and regular Ursodeoxycholic acid oral intake.

\section{Gallbladder puncture with needle}

A longitudinal echoendoscope (Pentax EG-3870-UT) with a working channel of $3.8 \mathrm{~mm}$ was introduced into the duodenal cavity to scan for the gall bladder and mark the puncture point. The contact zone (i.e., the region of the duodenal wall representing the shortest distance with gall bladder walls) was identified. Color Doppler was then used to identify interposing vessels in order to avoid them during puncture. An EchoTip Ultra endoscopic ultrasound needle (19-gauge, Boston Scientific Corp, Marlborough, Mass, USA) was introduced via the working channel of the echoendoscope, and the gallbladder was punctured under EUS guidance. A sample was aspirated to confirm that the punctured structure was gallbladder. The GB juice was amptied and refilled with saline and the contrast agent for cholecystography.

\section{Guidewire guided stent deployment group}

After the needle puncture, several loops of a guidewire $(0.035 \mathrm{inch} / 480 \mathrm{~mm}$; Boston Scientific, Bloomington, Ind, USA) were inserted into the gallbladder, then the needle was removed. Under EUS and fluoroscope guidance, the stent was slowly deployed. When the distal end of the stent contact with the duodenal wall, start the electrocautery and gently push the stent through the duodenal wall and then gallbladder wall.

Under the fluoroscope surveillance release the stent until the distal flanges was completely open. Gentle traction was applied to pull the gallbladder wall close to the gastric wall. Then, under endoscopic surveillance, the remainder of the stent was deployed (or just release the remainder stent within the endoscope). EUS was used to confirm the position of the stent and rule out leakage. 


\section{Anchor assisted stent deployment group}

After the needle puncture, the retrievable anchor is then passed along the needle into the GB and engaged to anchor the GB. After needle withdrawal, the GB is pulled with the anchor (Figure 2) when the GB is punctured and drained using ECE-LAMS. After the procedure, the retrieval cord is pulled with a pair of forceps, so the direction of the anchor is changed and it can be easily removed (Figure 3).

Per-oral transgastric cholecystoscopic therapy (cholecystolithotomy or cholecyst polyps' resection)

When the fistula between the GI tract and the GB has formed after the cholecystostomy with ECE-LAMS, per-oral cholecystoscopy could be performed.

CT or X-ray is used to determine that the stent remained in place. The endoscope is advanced into the GB via the fistula formed by the stent. A stone basket is inserted into the gallbladder to retrieve the stones. The basket is withdrawn from the gallbladder, and the stones are discharged into the GI tract. After several deployments of the basket to remove stones, an endoscope is introduced into the gallbladder to check for remaining stones. For the polypus resection, a snare or APC can be used. The stent could be removed after the procedure or before the stone removing (Figure 4). The normal diet should be resumed 48 hours after the stent removed.

\section{Data analysis}

Statistical analyses were carried out with SPSS version 23.0 (SPSS Inc., Chicago, III,USA). Frequencies, percentages, means ( \pm standard deviation) and medians (range) were used, as appropriate, for descriptive analysis. For categorical variables comparisons between groups were performed with the Fisher exact test $\$ small sample less than $40 \rrbracket$. Continuous variables with normal distribution were analyzed with the Student $t$ test, whereas for those with abnormal distribution analysis was performed with the MannWhitney $\mathrm{U}$ test. All statistical testing was 2-sided.

\section{Results}

A total of 24 ( 15 female) patients with gallstones were received the transluminal Cholecystolithotomy(Figure.5). Mean age is $36.08 \pm 13.69$ years old, ranging from 17 to 76 years old. The average diameter of the GB stones found in the ultrasound was $9 \pm 5.575 \mathrm{~mm}$, ranging from 4 to $21 \mathrm{mmm}$. 6 patients combined with common bile duct stones and 2 patients found combined with low confluence of cystic duct (Table1)..

\section{EUS-GB procedure}

The EUS-GBD was successfully performed in 20 (83.3\%) patients. The stent access route was through the duodenum in 21 (87.5\%) patients and stomach in $3(12.5 \%)$. The mean duration of the entire procedure 
was $19.65 \pm 3.554$ (range 8-26) min. Stent minor migration was found 14 days after the procedure in one patient and considered as the late stage adverse event. The fistula between GB and duodenal wall was still formed regardless the minor stent migration. No other sever adverse event was observed.

The guidewire assisted procedure was performed in 10 (41.7\%) patients, and retrievable anchor assisted procedure was performed in 14 (58.3\%). The successful rate of the anchor assisted group was high than the guidewire assisted group $(92.9 \% \mathrm{Vs} 70 \%, \mathrm{P}=0.07)$. The adverse event rate was similar between two groups. (Table 2)

\section{Per-oral cholecystoscopy}

On the average of $12.25 \pm 2.77$ (range 8-17) days after the initial BD drainage procedure, 20 patients received the per-oral cholecystoscopy via the fistula. The technique successful rate of per-oral cholecystoscopy techniqeus was $100 \%$. 15/20 patients completed the cholecystolithotomy, stones spontaneous passed outside the gallbladder was found in the remaining 5 patients. During the cholecystolithotomy, lithotripsy was performed in $3 / 15$ patients. GB polypectomy was performed in $5 / 20$ patients. The pathological results of the polyps are Cholesterol polyps in 3 patients, adenomas polyp in 1 and inflammation polyp in 1. During the cholecystolithotomy procedure, direct fistula tearing (injured by the introduce of the endoscope) was seen in one case (8 days post the GBD), which was repaired by metal clips. Contrast agent leakage (9days post the GBD) via the fistula was seen in another case during the balloon assisted gallbladder radiography, so the indwelling time of the stent was prolonged for $48 \mathrm{~h}$. No symptom was observed in this patient post the procedure. (Table 3 )

\section{Follow-up}

During the follow-up period (mean $314.2 \pm 213.7$, from 675.0 to 28.0 days) no GB stones recurrence or other long term complications reported.

\section{Discussion}

For the EUS-GBD techniqeu, it has undergone continuous improvement through the use of plastic stents, SEMS, and LAMS, these continuous improvements increase the successful rate ${ }^{6,7}$. However, the stenting procedure is skill challenging and time consuming. The newly developed ECE-LAMS has extremely shortened the operation time and reduce the bile leakage ${ }^{8,9}$. The large amount of the bile leakage will cause the gallbladder collapse during procedure and obvious sign of peritoneal irritation post the procedure. The total successful rate of EUS-GBD with ECE-LMPS in our study was $83.3 \%$ which is similar with other studies. Four (3 in the guidewire assisted group; 1 in the retrievable anchor assisted group) patients failed in the ECE-LAMS were all directly changed into the endoscopic trans-duodenal gallbladderpreserving cholecystolithotomy procedure, which has also been an available method ${ }^{10}$. No sever complications were observed in our study. 
In the early stage of our applications of EUS-GBD, we use the guidewire to facilitate the stent introduce, as reported by other studies ${ }^{11}$, which was high skill demanded and with the successful rate of $70 \%$. From 2017, we tried the RA to pre-inserted into the gallbladder, which would hold the GB closed to the duodenal wall and stabled the GB during the stent introducing. The use of the RA has increased the technique successful rate of EUS-GBD to $92.9 \%$. In most patients, ECE-LAMS can be easily implanted with the RA. We assumed that the RA has several advantages. First the anchored GB is more stable for the stent to target. Second, bile leakage may also be reduced because the puncture site was pressed by the duodenal wall. Third, In the stent release process, pulling the gallbladder can make the internal space larger, which is more conducive to reduce the probability of stenting failure. Of note, these anchors are non-damaging to the gallbladder, and they are retrievable. This method heightens the probability of success by ensuring a simpler and safer procedure. Operator confidence is boosted as well.

Formation of fistulous tract after EUS-GBD facilitates the entry of endoscope inside GB to perform endoscopic lithotomy and polypectomy. The average stent indwelling time before the transmural cholecystolithotomy is $12.25 \pm 2.77$ days. The longer stent indwelling time may have potential risks like migration which could be treated with LAMS-in-LAMS technique ${ }^{12}$. the shorter time duration may not be enough for the formation of fistulous tract and increase the chance of fistula broken. The contrast agent leakage was found in one case, when the attempted transduodenal cholecystolithotomy was perform 9 days after the EUS-GBD. Another case of fistula tearing was also occured during the transduodenal cholecystolithotomy, it was also 9 days after the GB stent. So, the stent indwelling time less than 10 days is not safe for the transmural cholecystolithotomy approach.

Gallstones removal in the GB is a simple procedure and lithotripsy could also be efficient in the treating large stone. In our study, $10 \%$ of the small stone can pass through the LAMPS spontaneously. Higher spontaneous stone passage was reported by other experts. The overall successful rate of transmural cholecystolithotomine our study is $100 \%$. The polypectomy was also successfully performed with the standard snare in 5 patients during the mucosal examination, which indicated the application of transmural cholecystoscopy ${ }^{13-15}$. The applications were also reported in other studies ${ }^{16}$.

The GB stones recurrence may be another important issue needs long term observation studies. It has been 4 years since the first EUS- transmural Cholecystolithotomy applied in our center, no stone recurrence was reported during the periodical follow-up. The patients are follow the protocol of Minimally invasive endoscopic gallbladder preserved cholecystolithotomy guideline in China (2015) If the GB stones recurred with symptoms, EUS- transmural Cholecystolithotomy could be repeated or the patients could still be considered as a surgical (eg. Laparoscopic cholecystectomy) candidate.

Our study implies that EUS based cholecystolithotomy is an efficient and minimally-invasive, scarless procedure which advanced in preserve the gallbladder and its related digestive functions. This has the potential to become the procedure of choice for gallstones besides LC. Long-term follow-up studies have found that cholecystectomy is associated with an increased incidence of dyspepsia, calculus of the common bile duct, and colon carcinoma ${ }^{17}$. So, LC at young patients could become a potential risk for a 
life long time. Gallbladder-preserving cholecystolithotomy could also be accomplished by laparoscope combined with choledochoscope, which has successfully preserve the GB function, and effectively avoid the various complications of cholecystectomy. During their follow-up, GB function was not affected and the stones recurrence rate was also quite low ${ }^{18}$.

There are a few limitations of our study. The study sample size is relatively small and prospective multicenter study design was needed in the future. There is still other endoscopic approach to treat gallstones, like endoscopic transmural gallbladder-preserving cholecystolithotomy procedure or endoscopic transmural cholecystectom. The comparison among these methods needs to be verified in the future.

\section{Conclusion}

In conclusion, we have demonstrated that EUS-assisted per-oral cholecystoscopy technique with ECELMAS is an efficient and safe procedure. For the EUS-GBD procedure, retrievable anchor could significantly increase the success rate compared with the guidewire ( $92.9 \% \mathrm{VS} 70 \%, \mathrm{P}<0.05)$. The stent indwelling time should no less than 10 days before the following endoscopic trans-mural operations. This conclusion is still need to be further proved by the long term multicenter prospective studies.

\section{Abbreviations}

EUS-GBD: endoscopic ultrasound guided gallbladder drainage

LAMS: lumen apposing metal stent

ECE-LAMS: electrocautery-enhanced delivery of LAMS

\section{Declarations}

Ethics approval and consent to participate:

The informed consent obtained was written. The protocol to perform retrospective revision of the cases was approved by the Medical Ethics Committees of Shengjing Hospital. 015PS16J

Consent for publication: Not Applicable

Availability of data and material:

The datasets used and/or analysed during the current study available from the corresponding manuscript.

Competing Interest:

No conflict of interest has been declared by N Ge, J Hu, F Yang, F Yang $₫ K$ Zhang and S Sun. 
Author's Contributions:

NG and SS in planning the study and drafting the manuscript; JH, FY and KZ in collecting the data; FY in interpreting the data. They all approved the final draft submitted.

Funding:

This study was supported by the project of Technology engineering research center of Liaoning Province (Grant No.2018225110). Natural Science Fund of Liaoning Province (Grant No. 20180530014)

Acknowledgements:

Not Applicable

\section{References}

1. James TW, Baron TH. Converting percutaneous gallbladder drainage to internal drainage using EUSguided therapy: A review of current practices and procedures. Endosc Ultrasound 2018;7:93-96.

2. Ge N, Wang Z, Sun S, et al. EUS assisted transmural cholecystogastrostomy fistula creation as a bridge for endoscopic internal gallbladder therapy using a novel fully covered metal stent. BMC Gastroenterol 2014;14:164.

3. Ge N, Wang S, Wang S, et al. Endoscopic ultrasound-assisted cholecystogastrostomy by a novel fully covered metal stent for the treatment of gallbladder stones. Endosc Ultrasound 2015;4:152-5.

4. Ge N, Sun S, Sun S, et al. Endoscopic ultrasound-assisted transmural cholecystoduodenostomy or cholecystogastrostomy as a bridge for per-oral cholecystoscopy therapy using double-flanged fully covered metal stent. BMC Gastroenterol 2016;16:9.

5. Zhang K, Sun S, Guo J, et al. Retrievable puncture anchor traction method for EUS-guided gallbladder drainage: a porcine study. Gastrointest Endosc 2018;88:957-963.

6. Small AJ, Irani S. EUS-guided gallbladder drainage vs. percutaneous gallbladder drainage. Endosc Ultrasound 2018;7:89-92.

7. Saumoy M, Novikov A, Kahaleh M. Long-term outcomes after EUS-guided gallbladder drainage. Endosc Ultrasound 2018;7:97-101.

8. Itoi T, Tsuchiya T, Sofuni A, et al. Development of EUS-guided gallbladder drainage and current indications. Endosc Ultrasound 2018;7:76-78.

9. Jain D, Bhandari BS, Agrawal N, et al. Endoscopic Ultrasound-Guided Gallbladder Drainage Using a Lumen-Apposing Metal Stent for Acute Cholecystitis: A Systematic Review. Clin Endosc 2018;51:450-462. 
10. Liu B, Du B, Pan Y. Video of the Month: Transrectal Gallbladder-Preserving Cholecystolithotomy via Pure Natural Orifice Transluminal Endoscopic Surgery: First Time in Humans. Am J Gastroenterol 2015;110:1655.

11. Oh D, Song TJ, Cho DH, et al. EUS-guided cholecystostomy versus endoscopic transpapillary cholecystostomy for acute cholecystitis in high-risk surgical patients. Gastrointest Endosc 2018.

12. Ligresti D, Cipolletta F, Amata M, et al. Buried lumen-apposing metal stent (LAMS) following endoscopic ultrasound-guided gallbladder drainage: the LAMS-in-LAMS rescue treatment. Endoscopy 2018;50:822-823.

13. Chan JHY, Teoh AYB. The development of peroral cholecystoscopy and advanced gallbladder interventions. Endosc Ultrasound 2018;7:85-88.

14. Adler DG. EUS-guided gallbladder drainage: Current status and future prospects. Endosc Ultrasound 2018;7:1-3.

15. Chen SA, Feng ZN, Li S, et al. EUS-guided gallbladder polyp resection: A new method for treatment of gallbladder polyps. Hepatobiliary Pancreat Dis Int 2018.

16. Tian L, Yang Y, Xiao D, et al. Resection of gallbladder polyps following endoscopic ultrasound-guided cholecystoduodenostomy using a lumen-apposing metal stent. Endoscopy 2018;50:E307-e308.

17. Carrilho-Ribeiro L, Serra D, Pinto-Correia A, et al. Quality of life after cholecystectomy and after successful lithotripsy for gallbladder stones: a matched-pairs comparison. Eur J Gastroenterol Hepatol 2002;14:741-4.

18. Tan YY, Zhao G, Wang D, et al. A new strategy of minimally invasive surgery for cholecystolithiasis: calculi removal and gallbladder preservation. Dig Surg 2013;30:466-71.

\section{Figure Legend}

Figure 1. The retrievable puncture anchor. The soft white string is the retrieval cord.

Figure 2. a.After the needle puncture, the retrievable anchor is then passed along the needle into the GB. b..After needle withdrawal, the GB is pulled with the anchor in the EUS Image.

Figure 3. a.The endoscopic view of the retrieval cord. b. The anchor is removed by pulling the retrieval cord by the forceps.

Figure 4. a.A stone basket is inserted into the gallbladder to retrieve the stones b. The endoscopic view of fistula after stent removal.

Figure $5 \otimes$ The chart flow of the patients selection and procedure 


\section{Figures}

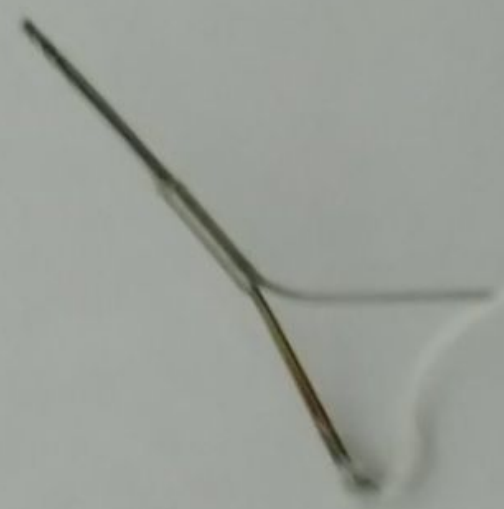

\section{Figure 1}

The retrievable puncture anchor. The soft white string is the retrieval cord.

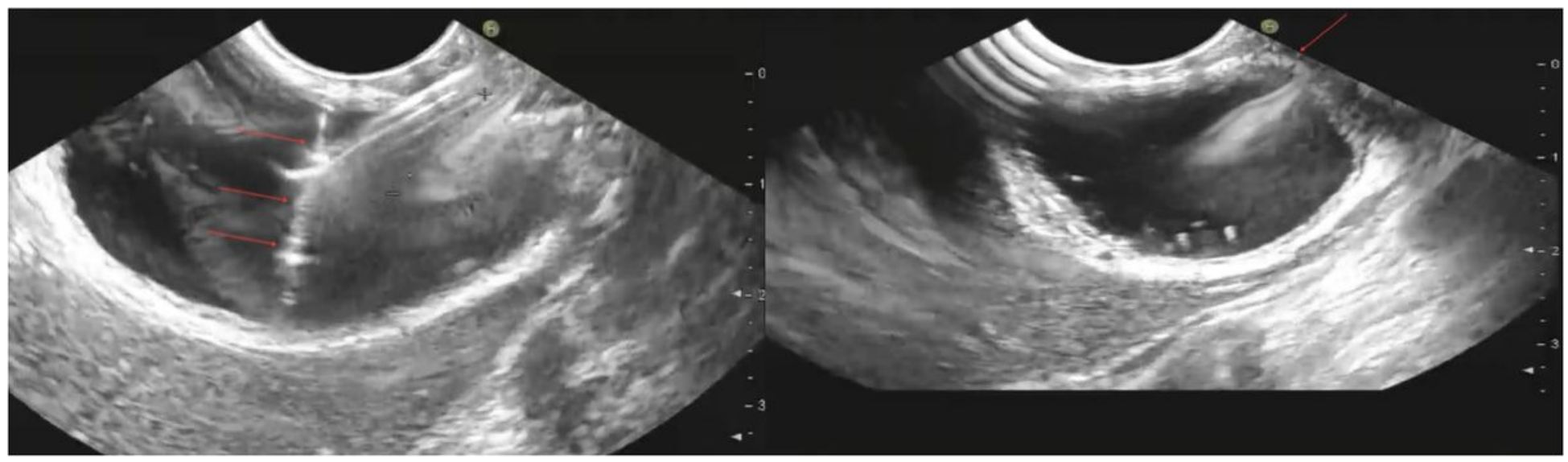

Figure 2 
a.After the needle puncture, the retrievable anchor is then passed along the needle into the GB. b..After needle withdrawal, the GB is pulled with the anchor in the EUS Image.

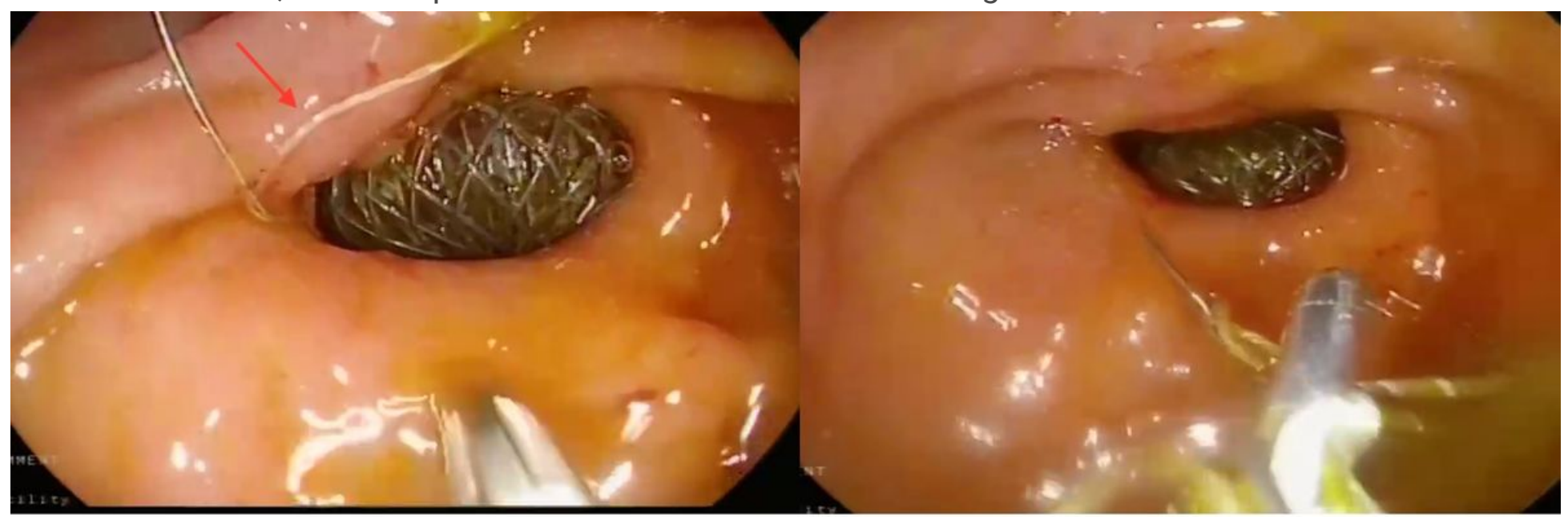

\section{Figure 3}

a.The endoscopic view of the retrieval cord. $b$. The anchor is removed by pulling the retrieval cord by the forceps.

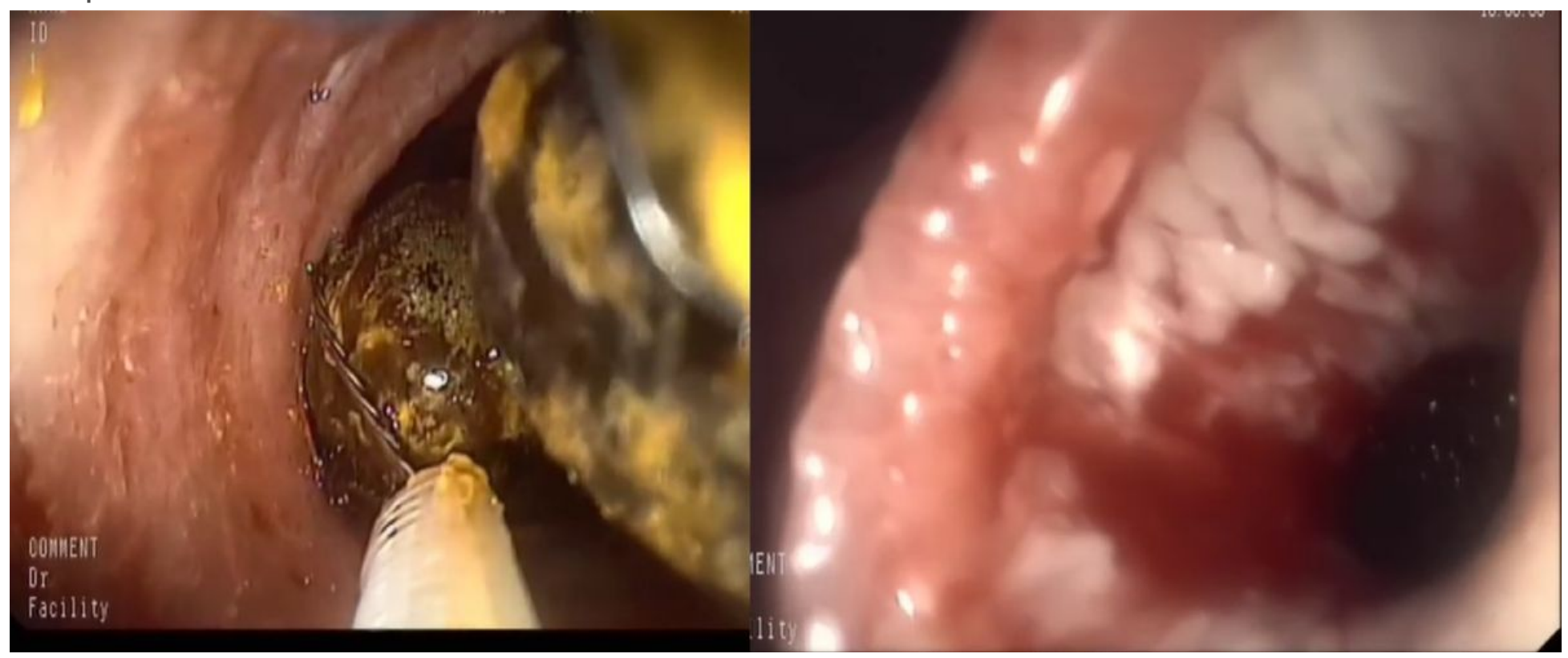

\section{Figure 4}

a.A stone basket is inserted into the gallbladder to retrieve the stones b.The endoscopic view of fistula after stent removal. 


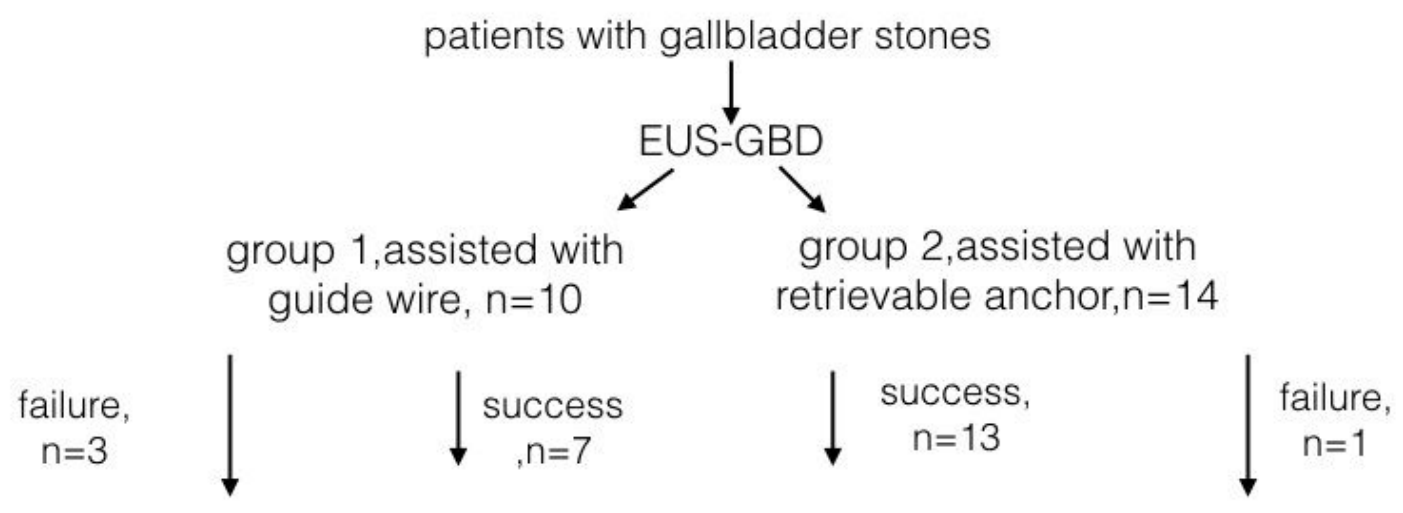

endoscopic transmural GB cut open and stone removing stent indwell and fistula forming, $\mathrm{n}=20$

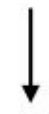

endoscopic transmural cholecystolithotomy or pylpectomy

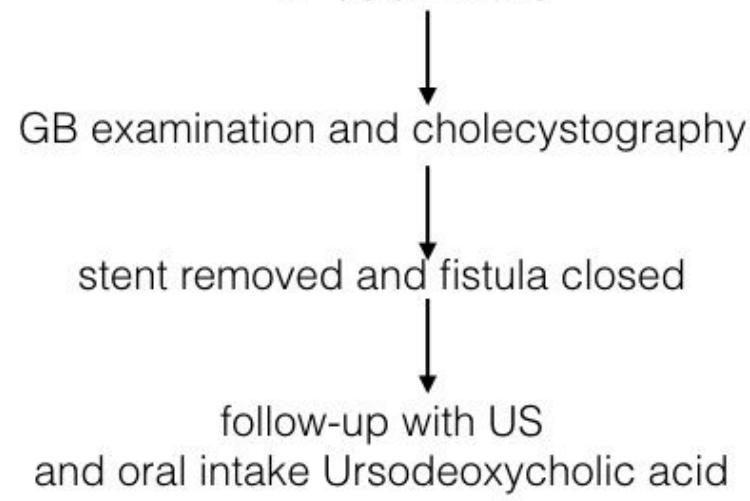

endoscopic transmural GB cut open and stone removing

\section{Figure 5}

The chart flow of the patients selection and procedure

\section{Supplementary Files}

This is a list of supplementary files associated with this preprint. Click to download.

- supplement1.pdf

- supplement2.pdf

- supplement3.pdf 